\title{
QTL mapping and loci dissection for leaf epicuticular wax load and canopy temperature depression and their association with QTL for staygreen in Sorghum bicolor under stress
}

\author{
H. O. Awika · D. B. Hays · J. E. Mullet • W. L. Rooney • B. D. Weers
}

Received: 1 February 2017/Accepted: 2 August 2017/Published online: 16 August 2017

(C) The Author(s) 2017. This article is an open access publication

\begin{abstract}
Plant waxes and staygreen are distinct phenotypic traits that have been independently implicated in heat and drought tolerance among grasses. The association between these two traits has not been fully explored, which makes the exploitation of synergy between them difficult. This study assessed the association between QTL regulating the staygreen (Stg) trait in sorghum and those regulating epicuticular wax load (WL) and associated canopy temperature depression (TD). Using a recombinant inbred line (RIL) population derived from Tx642 and Tx7000, phenotypic data were collected in three replicated field trials and one greenhouse trial. High absolute TD generally corresponded to high WL. The $\mathrm{r}^{2}$ of TD against WL was highest under non-stress conditions in the greenhouse while it was much larger in the cooler
\end{abstract}

H. O. Awika ( $₫)$ · D. B. Hays · W. L. Rooney

Soil and Crop Sciences Department, Texas A\&M

University, College Station, Texas, USA

e-mail: awik1@tamu.edu

D. B. Hays

Molecular and Environmental Plant Sciences, Texas

A\&M University, College Station, Texas, USA

J. E. Mullet · B. D. Weers

Deparment of Biochemistry and Biophysics, Texas A\&M

University, College Station, Texas, USA

J. E. Mullet

Institute for Plant Genomics and Biotechnology, Texas

A\&M University, College Station, Texas, USA and irrigated field conditions compared to hotter, drier field trials. The genetic correlations between the two traits also followed this pattern. Composite interval mapping identified a total of 28 QTL, 15 of which had significant overlaps between different traits. Most of the wax QTL were associated with pre-anthesis drought tolerant Tx7000. However, one QTL for WL overlapped with a QTL for staygreen (Stg2) and was represented by a single, isolated marker near the centromeric region on the short arm of SBI-01. The marker is identified by a Cis-acting regulatory module and is part of a 2-kb multifunctional motif-rich region which includes core promoter and enhancer regions and transcription elements, including a droughtresponsive MYB binding site. We suggest that this QTL may be pleiotropic for important stress tolerance mechanisms regulating both staygreen and leaf wax in sorghum.

Keywords Ab initio prediction - Canopy temperature depression - Cis-acting regulatory module $\cdot$ Epicuticular wax $\cdot$ Sorghum bicolor . Staygreen $\cdot$ Stress

\section{Introduction}

Drought is a major limiting factor in crop productivity. The capability of a plant leaf to sustain a healthy internal temperature in the face of erratic external 
conditions is vital in maintaining leaf cell water balance, and necessary for optimal flowering, seed development, and biomass accumulation (McLaughlin and Boyer 2004; Kumari et al. 2007). Adequate water availability and slowed post-anthesis senescence (staygreen) have been shown to have a direct positive impact on yield, while leaf epicuticular wax has been shown to deter excessive water loss through the leaf cuticle under elevated air temperatures and water deficit (Clarke and Richards 1988). Investigating the genetic association between staygreen and plant surface waxes might shed light into the possible interaction of the two traits in alleviating the impacts of stress on crops.

In cereal crops like grain sorghum, the staygreen trait has traditionally been defined as the ability of a plant to maintain active photosynthetic processes under water deficit, especially post anthesis (Crasta et al. 1999). When defined in terms of green leaf area, staygreen has been scored as a leaf death count (Xu et al. 2000) and more recently, leaf area under decline (LAUD) (Kumari et al. 2007), compared to a reference genotype (Thomas and Howarth 2000). This corresponds to the ability of a plant to maintain active photosynthetic processes with delayed senescence post-anthesis. In sorghum, staygreen has been associated with QTL that are stable across environments (Tao et al. 2000; Haussmann et al. 2002; Kassahun et al. 2010). In general, four major QTL for staygreen individually exhibit different tolerance to droughtinduced senescence (Harris et al. 2007; Kassahun et al. 2010; Xu et al. 2000; Rosenthal and Gerik 1991), as defined by the ability of some sorghum genotypes to stay green during post-anthesis water stress (Blum 2005; Tao et al. 2000).

The induction of senescence involves a complex regulatory network in leaf senescence processes (He et al. 2002; Lim and Nam 2005; Lim et al. 2003; Noh and Amasino 1999; Quirino et al. 2000). Some senescence up-regulated transcription factor genes belong to various transcription factor families including SARK (senescence-associated receptor kinase), GTP-binding protein, WRKY (WRKY amino acid signature-containing DNA-binding domain), EREBP (ethylene responsive elements binding protein), NAC (no apical meristem), and MYB (myeloblastoma) families (Quirino et al. 2000; Yoshida et al. 2002). Of these, the genes for WRKY53, a MYB protein, and zinc finger protein show transiently increased expression at a very early stage of leaf senescence (Buchanan-Wollaston et al. 2003; Guterman et al. 2003; Hinderhofer and Zentgraf 2001).

Because wax modulates canopy temperature and buffers against abiotic stress-induced injury and signal transduction, it may be important in maintaining the functionality of staygreen. Waxes thus may reduce stress-related stimuli for catabolic processes, thereby slowing natural aging and degradation of chlorophyll at the height of reproductive activity. The onset of wax accumulation is well known to be at early vegetative phases of many grass species (Clarke and Richards 1988). The synchrony of its peaking during florescence and anthesis in some plant species suggests a possible association with constitutive genetic control of staygreen and improved tolerance to heat and drought among tolerant variants. In this study, we have begun to test this relationship and the genetic association between the two traits.

\section{Materials and methods}

Germplasm, mapping population and evaluation environments

One hundred $F_{12}$ recombinant inbred lines (RILs) developed from a cross of Tx642 and Tx7000 were used in this study. Tx642 (B35) has been a useful source of staygreen for research and development of drought tolerant hybrids (Harris et al. 2007; Tao et al. 2000). It is an inbred $\mathrm{BC}_{1}$ derivative line of IS12555 durra sorghum from Ethiopia, and varying dominance is exhibited by its genes regulating the staygreen trait (Xu et al. 2000; Sanchez et al. 2002). Tx642 is susceptible to pre-flowering drought stress but highly tolerant to post-flowering drought stress (staygreen trait). Tx7000 is an elite line released by the Texas Agricultural Experiment Station at Lubbock. It was derived from a 'Kafir' $\times$ 'Milo' cross, and was originally grown as a cultivar and then later used as a male parent in the late 1950s (Xu et al. 2000). It is currently a public line used in breeding programs in the United States (Subudhi et al. 2000). Tx7000 is preflowering drought tolerant and post-flowering drought susceptible (Xu et al. 2000).

A total of four environments were used to phenotype epicuticular wax load (WL) and canopy temperature depression (TD). The environments consisted of 
three field trials at Texas A\&M AgriLife Research Centers in College Station (irrigated), Weslaco (irrigated) and Corpus Christi (rainfed) in 2011. A fourth evaluation environment was in the greenhouse at College Station with normal watering in 2011. Each RIL was sown as three replicates in each field environment, and three replicates (pots) with two plants per pot in the greenhouse.

The field trials were sown in the spring of 2011: on February 16th for Weslaco, March 9th for Corpus Christi and on March 23rd for College Station. All plots were sown in randomized complete block design (RCBD) with each experimental unit composed of two contiguous rows $5.3 \mathrm{~m}$ in length with $\sim 110-130$ plants per row. The spacing between rows was $76 \mathrm{~cm}$ in Weslaco and College Station, and $1.0 \mathrm{~m}$ in Corpus Christi. Open furrow irrigation was applied in Weslaco and College Station over the growth period, while Corpus Christi was rain fed 1 day after sowing. All other standard agronomic practices were conducted as typical for the region. Canopy temperature readings and leaf samples for wax analysis were taken at $50 \%$ flowering, observed independently for each plot.

In the greenhouse, 100 RILs were sown in $30 \mathrm{~cm}$ diameter pots filled with potting mix (MetroMix 900TM forest peat moss, Schulenburg, Texas), on December 4, 2011. The pots were watered one $d$ after sowing and at subsequent intervals of 4-6 days thereafter until flowering. No water was applied for the 2 days prior to each sampling date. Canopy temperature readings and leaf samples for wax analysis were taken on the same day at $50 \%$ anthesis on a plot basis. The mean air temperatures in the growth environments were taken to be the means averaged $\sim 13 \mathrm{~cm}$ above the canopies on a plot basis (or pot basis in the greenhouse) between 12:00 and 14:00 using an external air temperature probe component of a digital infrared thermometer (IRT, see next section). The temperatures during the reproductive phase through the sampling period were as follows: greenhouse, late February 2011: 24-26.5 ${ }^{\circ} \mathrm{C}$; Weslaco, early June 2011: $\sim 25-30{ }^{\circ} \mathrm{C}$; Corpus Christi, late June 2011: 39-41 ${ }^{\circ} \mathrm{C}$, and College Station, mid July 2011: 42-46 ${ }^{\circ} \mathrm{C}$.

\section{Phenotyping and design}

Days to flowering were recorded for each plot. At $50 \%$ anthesis on a plot basis in the field environments, four plants per replicate were randomly sampled and 10 fresh leaf disks, $7.0 \mathrm{~mm}$ in diameter, were punched from the flag leaf of each selected plant. The leaf disks were punched directly into clean vials, one vial per plant, using a handheld Precision Leaf Sampler, No. 11325 Series (IL, USA, www.rabbittool.com). All plants grown under greenhouse conditions were similarly sampled. A colorimetric method (Ebercon et al. 1977) was used to extract leaf epicuticular wax and the spectrometric absorbance was obtained at $290 \mathrm{~nm}$ using a PHERAStar FS spectrometer integrated with MARS Data Analysis Software V 3.10R3 (BMG LABTECH, Ortenberg, Germany; http://www. bmglabtech.com). Data were collected in late February 2011 (greenhouse), early June 2011 (Weslaco), late June 2011 (Corpus Christi) and mid July 2011 (College Station). The Beer-Lambert conversion algorithm (Paynter 1981) was used from a new standard curve to convert absorbance readings to wax load in $\mathrm{mg} \mathrm{dm}^{-2}$.

The timing for temperature sampling was during clear skies between 12:00 and 14:00 when the correlation of canopy temperature at anthesis and yield is highest (Balota et al. 2007a, b) and plant water stress is expected to be high (Balota et al. 2007a, b; Fan et al. 2005) for the stress-treated field environments. The leaf temperatures $\left(\mathrm{T}_{1}\right)$ for all plants were averaged using a hand-held IRT (model OAKTon Pro, Class 2(ii) laser product, output wavelength 630-670 nm, with external probe for ambient air temperature sensing). The incident laser point was beamed at an angular tilt according to leaf orientation such that it was incident $13 \mathrm{~cm}$ perpendicular to at least six different points on the target flag leaf. The external probe component of the IRT was simultaneously used to record the ambient air temperature $\left(\mathrm{T}_{e}\right)$. The difference $\left(T_{e}-T_{1}\right)$ was taken as the temperature depression (TD). For the field environments, each TD data point was the average of six readings per plant and 10 plants per RIL (five plants for each of the two replicates per RIL), including the plants that were randomly tagged for WL sampling. In the greenhouse, six readings were taken per plant for each of two plants per replicate. The TD readings were taken on the same day as the leaf wax but before leaf disks were taken. All the TD data were taken using the same IRT sensor to eliminate possible errors due to technical discrepancies between instruments. It should be noted that a population size of 100 RILs (and the two parents, for a 
total of 102 lines) was determined to be optimal for the 2-h-constrained TD sampling window, the sample size, sampling instrument and sampling rate as defined for this experiment. The 100 RILs were used in QTL mapping.

Determining the phenotypic variance components, heritability and genetic correlation

To estimate the variance components, we applied residual maximum likelihood (REML) modeling tool in $\mathrm{R}$ version Ri 386 3.2.0 ( $\mathrm{R}$ Core Team 2013). The REML method was chosen for its flexibility in analysis experiments involving fixed effects and random effects with no restriction to independent data, or to data with the same variance in any one stratum (O'Neill 2010; Hill et al. 2008). Environment, genotype and replicates were treated as random effects while WL and TD were the random response variables in the combined environment analysis. The resulting data were used to partition the phenotypic variance of each trait into genotypic variance, variance due to environment and the variance due to genotype by environment interaction. We estimated broad-sense heritability $\left(\mathrm{H}^{2}\right)$ on an entry mean basis (Bernardo 2002) for each trait in each environment. In each environment, we also determined the proportion of phenotypic correlation between WL and TD that is attributed to genetic causes (a measure of their genetic correlation, $r_{\mathrm{g}}$ ) (Maes et al. 2004) using the phenotypic variance and heritability components according to the model below (Falconer et al. 1996):

$r_{g}=\frac{r_{p}-e_{1} e_{2} r_{e}}{h_{1} h_{2}}$ and $r_{e}=\frac{\sigma_{e 12}^{2}}{\sigma_{e 1}^{2} \sigma_{e 2}^{2}}$,

where $r_{\mathrm{p}}$ is the phenotypic correlation between trait 1 (WL) and trait 2 (TD), derived as $r_{\mathrm{p}}=h_{1} h_{2} \cdot r_{\mathrm{g}}+$ $e_{1} e_{2} \cdot r_{\mathrm{e}} ; h_{1}$ and $h_{2}$ are the broad-sense heritability of trait 1 and trait 2, respectively; $e$ is the environmental variance and $r_{\mathrm{e}}$ is the non-zero environmental correlation between trait 1 and 2 . The symbol $\delta^{2}$ is the environmental variance on the traits.

The ratio of expected phenotypic correlation $\left(r_{\mathrm{p}}\right)$ to that of the observed $r_{\mathrm{p}}$ (Wachs and Plomin 1991) estimated the proportion of the observed phenotypic correlation between WL and TD that is due to genetics. Observed phenotypic correlation between WL and TD was derived from the coefficients of determination $\left(\mathrm{r}^{2}\right)$ between the two traits in each environment. Expected $r_{\mathrm{p}}$ was determined as a bivariate heritability, calculated as the square roots of heritability of the two traits multiplied by their genetic correlation $\left(\sqrt{ } h_{1} \cdot \sqrt{ } h_{2} \cdot r_{\mathrm{g}}\right)$, without the environmental correlation terms (Wachs and Plomin 1991; Waitt and Levin 1998).

Statistical transformation for QTL analysis

Fluctuations in air temperatures within a sampling window were noted to be significant on some days. To reduce the error of variation due to this, the TDs were moderated following recommendations in previous studies (Keener and Kircher 1983; Reynolds et al. 1994, 1998; Wilman et al. 1992; Scott and Knott 1974). First, the minimum and the maximum ambient air temperatures were noted for each sampling day during the 12:00-14:00 sampling window. This data was used to cluster the recorded air temperatures $\left(T_{e}\right)$ within a day's sampling window into bin widths of $0.5{ }^{\circ} \mathrm{C}$. The mean TD for each bin (cluster) was noted. The mean TD from 6 readings per plant was first divided by the mean TD of the representative cluster and then multiplied by the mean TD per plant to obtain a corrected TD value for the plant. This ensured minimal deviation from the actual raw TD, while achieving the versatility required to reduce the error variance (Kaufman and Rousseeuw 2009) associated with instantaneous ambient temperature fluctuations within a day's data collection window. The corrected TD correlated positively $(r=0.94)$ with the raw TD data, and was applied in the subsequent regression analyses. Log-transform values of WL and the raw data for number of days to $50 \%$ flowering (FT) were also used. The effect of FT is reported only in the QTL analysis.

The homogeneity of phenotypic variance among locations was determined using the Lavene Test as modified by Brown-Forsythe (Ott and Longnecker 2010), and the normality assumption was checked by the Shapiro-Wilk Test (Ott and Longnecker 2010). A generalized linear model (GLM) was used to assess the model fitness and the coefficient of determination $\left(\mathrm{r}^{2}\right)$ of TD as explained by WL. For the non-QTL analysis, the mean separation was determined using Fisher's LSD. Regression fits were performed in JMP Pro 12 (SAS) and bar graphs in MS Excel. 
QTL mapping and analysis

Digital genotyping (DG) (Morishige et al. 2013) was accomplished using the high throughput Illumina Genome Analyzer Platform II (Illumina.com 2014), a next generation sequencer previously described (Quail et al. 2001). The Illumina sequencer is hosted at the Texas A\&M AgriLife Genomics and Bioinformatics Services in College Station, Texas, USA. The genotyping data was processed from BTx642/ RTx7000 RIL population genomic DNA digested by NgoMIV. A total of 886 DG markers were used in this QTL study. MapMaker/EXP version 3.0 (Stephen 1993) was used to create the genetic map.

The phenotypic values for WL and TD were averaged per RIL across reps to produce100 sample values per trait in each location used for QTL analysis. WinQTL Cartographer V2.5 (Wang et al. 2011) was used to generate QTLs using composite interval mapping at a walk speed of $1 \mathrm{cM}$. For each trait, the data were permuted 1000 times to establish the QTL significance threshold. QTL above the 50th rank (95\%) were deemed significant. QTL peak position, 1-LOD widths, and the proportion of phenotypic variance explained were extracted from the plots. All QTL were overlaid on the Stg QTL positions previously published (Crasta et al. 1999; Subudhi et al. 2000; Xu et al. 2000) for the same mapping population. These previously mapped Stg loci were derived from a visual phenotypic rating on a to 5 scale. A value of 1 corresponded no leaf senescence, and 5 represented total leaf senescence at grain maturity. Using similar scoring, other studies have confirmed and identified additional loci (Harris et al. 2007; Kassahun et al. 2010; Sanchez et al. 2002) for the staygreen trait in other regions of the sorghum genome. MapChart V2.2 (Voorrips 2002) graphical interface was used to organize the QTL bands on the linkage graphs obtained from WinQTL Cartographer V2.5.

Identifying annotated genes under overlapping QTL for wax and QTL for staygreen

A primary interest in this study was the overlap of QTL for wax and staygreen; therefore, only the sequences in such regions of significant overlap were searched in detail. For the sake of this study, the marker coinciding with a QTL peak has been termed 'peak marker'. The digital genotyping (DG) QTL peak markers at 1-LOD were taken to represent the position of the respective QTL. The range flanked by the vertical intersects to a QTL trace line (curve) on either side of the peak marker was taken as the QTL width. Each DG marker under a QTL is distinguished by a unique short genome sequence acquired from the polymorphic DG templates. The limits of the genomic sequence bound by a declared QTL were defined by the start nucleotide of the first marker and the end nucleotide of the last marker flanking a peak marker, depending on the read orientation (reverse or forward) of each marker sequence.

For QTL with distinct flanking markers, the genomic coordinates of the QTL were searched and viewed at the 'Pairwise Level' in the 'Genome Context' of the sorghum portal of the Plant Genome Database (PGDB), home browser SbGDB (Sorghum bicolor GDB) assembly version Sbi1/Sbi1.4/ SbGDB181 (http://plantgdb.org/SbGDB/). Using the Zoom toggle in SbGDB Genome Context Tool, the subject chromosome was walked at $5 \mathrm{~kb}$ per toggle beginning with the $5^{\prime}$ marker sequence until the end of the QTL boundaries. Annotated transcripts in this region were confirmed and noted. All sequences were downloaded in FASTA format.

Where an uncharacterized transcript was identified, its CDS (coding DNA sequence) was first queried in the NCBI's BLASX (Basic Local Alignment and Search Tool for nucleotide to protein) (Stephen et al. 1997) context for orthologous protein translations. The search was refined in Phytozome V.10 (The Plant Comparative Genomics portal of the Department of Energy's Joint Genome Institute; phytozome.jgi.doe.gov), BLASTX for the grasses node. The top eight BLASTX hits of non-redundant transcripts with small E-values below the threshold and scores greater than the threshold value were selected for multiple sequence alignment (MSA) in the UniProt platform (Consortium 2014) and their relatedness was ascertained through using CLUSTALW (Kyoto University Bioinformatics Center; http://www.genome.jp/tools/ clustalw/) to construct a phylogenetic tree. The amino acid (AA) residue sequence of an annotated subject polypeptide which shared a phylogenetic node closest to the sorghum sequence was reported as the representative putative/characterized transcript. The annotation and gene ontology for this selected transcript were obtained in the UniProt Knowledge Base (UniProtKB/Swiss-Prot), a manually annotated and non- 
redundant protein sequence database. The NCBI's DELTA-BLAST (domain enhanced lookup time accelerated-BLAST) was used for an improved identification of conserved base pair level functional domain, and the domain family tree noted in Pfam (Protein Families, http://pfam.xfam.org/) and InterPro of the European Molecular and Bioinformatics Lab, EMBL, http://www.ebi.ac.uk/interpro).

$\mathrm{Ab}$ initio prediction of the non-exomic function of non-annotated marker sequences

A marker sequence resident in an annotated transcript was noted as representing that transcript. In the case where no flanking markers were available or where a peak marker did not directly correspond to an exome region, the search was zoomed out to $1 \mathrm{~kb}$ immediately upstream and $1 \mathrm{~kb}$ immediately downstream of the peak marker sequences. The $1 \mathrm{~kb}$ region is within many of the new chromatin immunoprecipitation, ChIP-derived data of DNA segments containing nonexome functional elements (Worsley-Hunt et al. 2011). These were used in the first ab initio gene prediction in Genefinder in the FGENESH suit (Solovyev 2001; Solovyev et al. 2006; Yao et al. 2005) which applies the HMM (hidden Markov model) algorithm (Stratonovich 1960; Baum and Petrie 1966). HMM is used to find model parameters that best fit the observations given a model and empirical observations. Where no genes were predicted a second ab initio search was conducted for the discovery of Cis-acting regulatory modules in PlantCARE (Plant $\mathrm{Cis}$-acting regulatory elements) (Magali Lescot et al. 2002) and confirmed in ESEfinder (exonic splicing enhancer finder) V3.0 (Smith et al. 2006; Cartegni et al. 2003).

\section{Results}

Relationship between epicuticular wax load and plant canopy temperature

The mean air temperature recorded $\sim 13 \mathrm{~cm}$ above the canopies and averaged for all the plots during flowering at College Station ranged between 41.5 and $46{ }^{\circ} \mathrm{C}$, the highest among field environments. Corpus Christi had a mean of $39-41{ }^{\circ} \mathrm{C}$ and Weslaco was the lowest at $25-30{ }^{\circ} \mathrm{C}$ and comparable to the greenhouse temperature of $24-26.5^{\circ} \mathrm{C}$. Among field environments, Corpus Christi had the highest correlation $\left(\mathrm{r}^{2}=0.46\right)$ between $\mathrm{WL}$ and TD of the canopy (Fig. 1) with smaller correlations in College Station $\left(r^{2}=0.33\right)$ and Weslaco $\left(r^{2}=0.03\right)$. In, comparison, the greenhouse conditions had the strongest correlation $\left(\mathrm{r}^{2}=0.83\right)$. These results, in part, support previous findings (Mondal et al. 2015) that leaf wax load is correlated with the ability of a plant to keep itself cooler through increased albedo, resulting in reduced water loss under elevated heat stress.

We further tested this relationship by estimating the genetic correlations $\left(r_{\mathrm{g}}\right)$ between WL and TD in the four environments. The strongest $r_{\mathrm{g}}(0.88)$ showed in the heat-stressed College Station, followed by Corpus Christi (drought-stressed, $r_{\mathrm{g}}=0.79$ ), with the weakest genetic correlation $\left(r_{\mathrm{g}}=0.17\right)$ in the cooler Weslaco (Table 1). An $r_{\mathrm{g}}=0.62$ was recorded between the two traits in the greenhouse. The proportion of the phenotypic correlation that was due to genetics was determined by the ratio of expected phenotypic correlation to the observed phenotypic correlation ( $\left.\operatorname{Exp} r_{\mathrm{p}} / \mathrm{Obs} r_{\mathrm{p}}\right)$, expressed as a percent. An estimated $57.1 \%$ of the Obs $r_{\mathrm{p}}$ in Corpus Christi was due to genetics, as were $\sim 67 \%$ of Obs $r_{\mathrm{p}}$ in College Station and $\sim 17 \%$ Obs $r_{\mathrm{p}}$ in Weslaco. Generally, across three of the four environments, the magnitude and trend of $r_{\mathrm{g}}$ did not correspond to those of Obs $r_{\mathrm{p}}$ but $r_{\mathrm{g}}$ corresponded near perfectly with $\operatorname{Exp} r_{\mathrm{p}} / \mathrm{Obs} r_{\mathrm{p}}$.

Broad-sense heritability $\left(\mathrm{H}^{2}\right)$ within an environment ranged between 0.49 and 0.63 for $\mathrm{WL}$ and between 0.29 to 0.74 for TD, with the largest $\mathrm{H}^{2}$ being reported in the greenhouse environment for both traits. In combined analysis of all study environments, $\mathrm{H}^{2}$ of WL was 0.65 while $\mathrm{H}^{2}$ of TD fell to 0.11 (Table 1). There were no statistical differences between the mean WL in the Corpus Christi and the mean WL in College Station; mean WL were significantly larger in both environments, compared to those in the cooler, irrigated greenhouse and Weslaco (Table 1). The variance components for genotype $(\mathrm{G})$ and genotype $\times$ environment $(\mathrm{G} \times \mathrm{E})$ were significantly higher than zero (the value zero was not included within $95 \%$ lower confidence limits and 95\% upper confidence limit) for both WL and TD (Table 1). The environment variance component was surprisingly not significant for WL and for TD, suggesting that the differences in the phenotypic means and in heritability across environments, and also in the phenotypic 

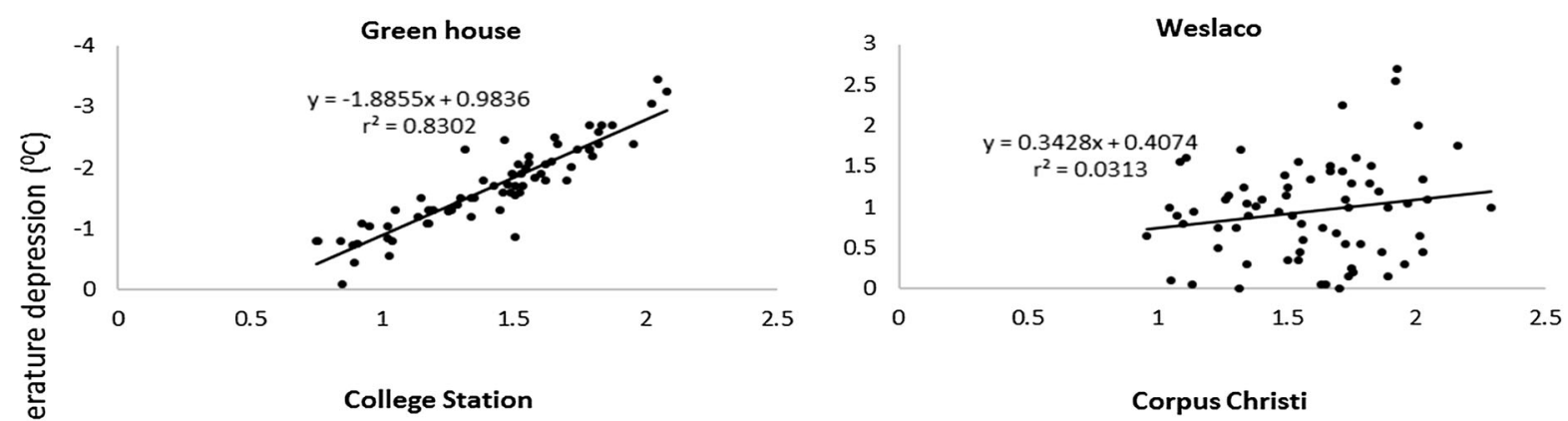

College Station

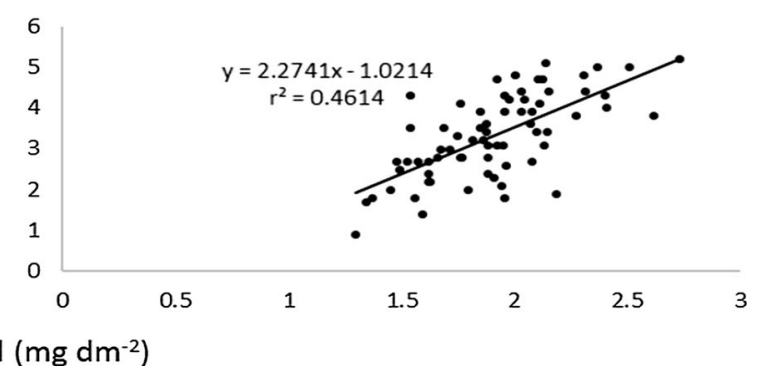

Fig. 1 Regression relationship between canopy temperature depression (TD) and epicuticular wax load (WL) in each location for the RIL population. On the Y-axis is TD adjusted for transient ambient air temperature $\left(\mathrm{T}_{\mathrm{e}}\right)$ within a measurement

correlation between WL and TD across the environments, were influenced mainly by genotype and $\mathrm{G} \times \mathrm{E}$ interactions.

\section{Pleiotropic QTL for wax}

A total of 28 QTL on 5 chromosomes were identified for canopy temperature depression (TD), epicuticular wax load (WL) and flowering time (FT). Most of the loci alleles associated with high values for wax and TD were contributed by the pre-anthesis drought tolerant parent, Tx7000. Alleles for extended flowering time were mainly contributed by the senescence resistant (staygreen) parent, Tx642. In the combined analysis 16 QTL were detected for TD, 9 QTL for WL and 3 QTL for FT (Tables 2, 3).

A number of QTL were overlapping for associated traits. On SBI-10, the QTL TDwl-10-58 explained $11.1 \%$ of phenotypic variance (PVE) for TD and it coincided with two QTL for leaf wax WLwl-10-58 $(\mathrm{PVE}=12.6 \%)$ and $W L c x-10-58(\mathrm{PVE}=13.2 \%)$, detected in Corpus Christi and Weslaco, respectively. These QTL were associated with the same marker, c10B13413, located at $108.2 \mathrm{cM}$. This suggests that the locus may be pleiotropic and conditional. A window (12:00-14:00). The canopy temperatures of all plants sampled in the greenhouse were warmer than the surrounding air temperatures, hence the negative sign on TD (vertical axis)

possible linkage between QTL for temperature depression and flowering time were also detected on other segments of SBI-10. For example, the QTL for TD (TDwl-10-33) was associated with marker c10B4064 at $62.6 \mathrm{cM}(\mathrm{PVE}=19.6 \%)$ and co-located with QTL FTcx-10-35 (also detected in a second environment) at marker c10F4552 (61.4 cM). On SBI-08, a QTL for TD, TDcx-8-15 (19.6\% PVE) and a QTL for FT, FTcx$8-15$ (11.7\% PVE), both centered at $55.8 \mathrm{cM}$, collocated with a QTL for wax, WLcx-8-17 (11.5\% PVE) positioned at $57.7 \mathrm{cM}$. These QTL were detected in the same environment (Corpus Christi), suggesting that a group of tightly linked genes at this locus affect these traits. On SBI-06, QTL for TD (TDcs-6-14) and one for WL (WLcs-6-14) were detected in the same field environment (College Station). Both were associated with the maker $c 6 B 8118$ located at position $46.8 \mathrm{cM}$. The PVE attributed to the QTL were 13.5 and $12.1 \%$, respectively. This further suggests a pleiotropic interaction between QTL for these two traits.

The Stg QTL that we have reported on SBI-01 and SBI-03 (Fig. 2) are the only ones which collocated with the other traits in this study. We did not identify any additional overlaps between the traits in this study 


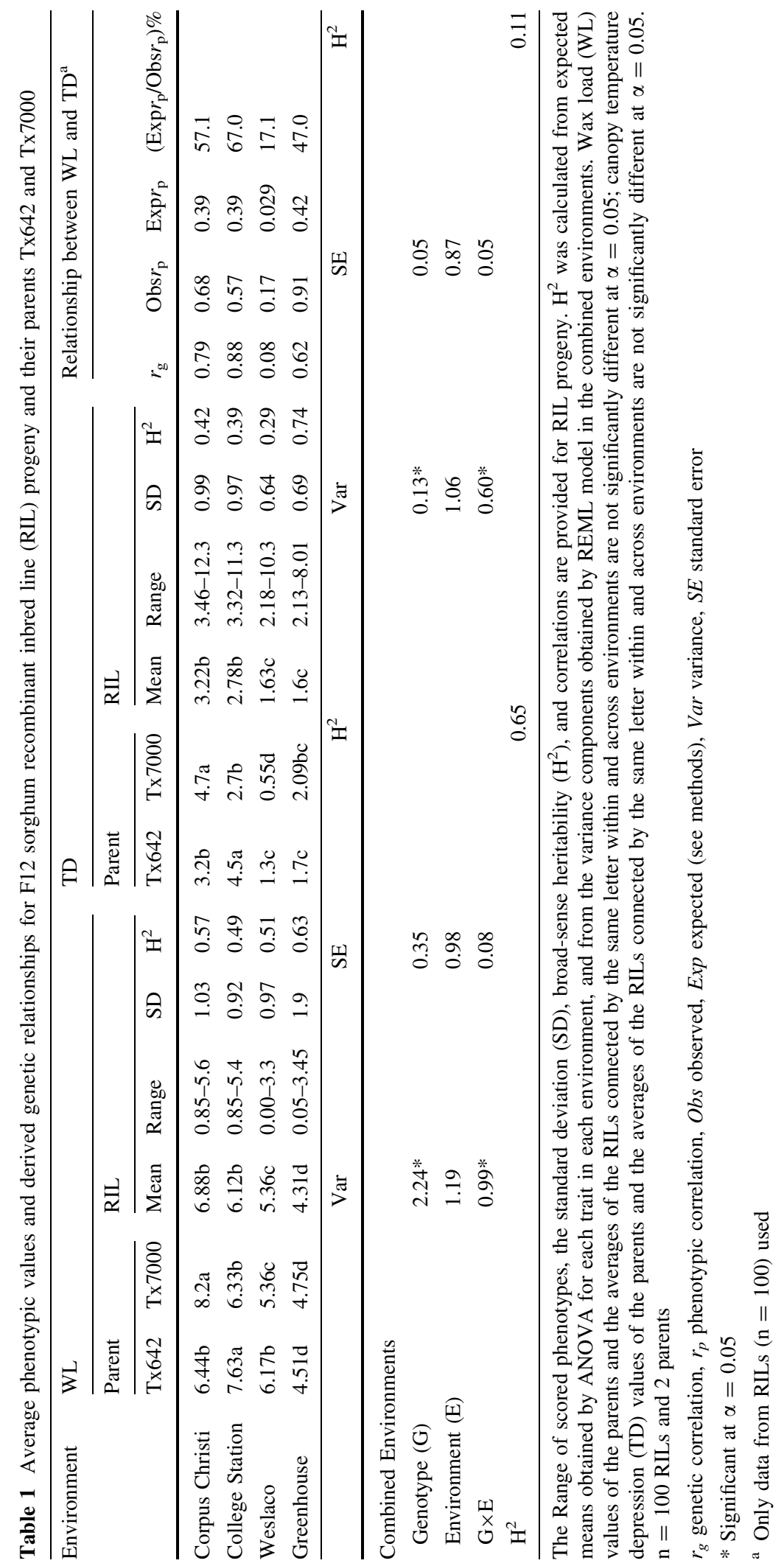


Table 2 QTL detected in BTx642/RTx7000 Mapping Population $(\mathrm{n}=100)$ in the greenhouse

\begin{tabular}{llllrlll}
\hline QTL $^{\mathrm{a}}$ & Markers & Position & LOD score & Additive & $\mathrm{R}^{2}$ & Positive allele & Environment detected \\
\hline WLgh-3-86 & c3F19429 & 131 & & 0.1222 & 0.1049 & Tx7000 & Gh, Wl \\
TDgh-3-74 & c3B17049 & 104.6 & 2.6504 & -0.2204 & 0.0986 & Tx642 & Gh \\
TDgh-8-1 & c8B168 & 4 & 2.8813 & -0.2399 & 0.1132 & Tx642 & Gh \\
TDgh-8-4 & c8F787 & 11 & 3.3425 & -0.243 & 0.1136 & Tx642 & Gh \\
FTgh-1-29 & c1B5373 & 32.6 & 4.2071 & -0.2277 & 0.1403 & Tx642 & Gh \\
FTgh-10-35 & c10F4552 & 61.4 & 7.7535 & 8.3466 & 0.1572 & Tx7000 & Gh, Cx \\
\hline
\end{tabular}

a QTL symbols: the first two upper case letters are the abbreviated traits ( $W L$ wax load, $T D$ canopy temperature depression, $F T$ flowering); the lower-case letters represent location ( $g h$ greenhouse); middle number is the chromosome number, and the last number in the marker number

Environment detected: $C x$ Corpus Christi, $G h$ greenhouse, $W l$ Weslaco

Table 3 QTL detected in BTx642/RTx7000 Mapping Population $(\mathrm{n}=100)$ in the field

\begin{tabular}{|c|c|c|c|c|c|c|c|}
\hline QTL $^{\mathrm{a}}$ & Markers & Position & $\begin{array}{l}\text { LOD } \\
\text { score }\end{array}$ & Additive & $\mathrm{R}^{2}$ & $\begin{array}{l}\text { Positive } \\
\text { allele }\end{array}$ & $\begin{array}{l}\text { Environment } \\
\text { detected }\end{array}$ \\
\hline \multicolumn{8}{|c|}{ Leaf cuticular wax } \\
\hline WLcs-1-32 & $\mathrm{c} 1 \mathrm{~F} 5510$ & 38.4 & 3.2668 & -0.147 & 0.1293 & Tx642 & $\mathrm{Cs}, \mathrm{Cx}$ \\
\hline WLcs-6-14 & c6B8118 & 47.2 & 3.4709 & -0.1434 & 0.1208 & Tх642 & $\mathrm{Cs}$ \\
\hline WLwl-3-86 & c3F19429 & 131 & 3.3304 & 0.1218 & 0.1097 & Tx7000 & Wl, Gh, \\
\hline WLwl-10-58 & c10B13413 & 107 & 3.4668 & 0.126 & 0.1267 & Tx7000 & $\mathrm{Wl}, \mathrm{Cx}$ \\
\hline WLcx-8-17 & c8B9861 & 57.7 & 3.1846 & 0.1219 & 0.1151 & Tx7000 & $\mathrm{Cx}, \mathrm{Wl}$ \\
\hline WLcx-10-50 & c10B12119 & 83.9 & 2.6205 & -0.1322 & 0.0917 & Тх642 & $\mathrm{Cx}$ \\
\hline WLcx-10-55 & c10B13167 & 99.9 & 2.8261 & 0.1401 & 0.1037 & Tx7000 & $\mathrm{Cx}$ \\
\hline WLcx-10-58 & c10B13413 & 108.2 & 3.6789 & 0.1397 & 0.1323 & Tx7000 & $\mathrm{Cx}, \mathrm{Wl}$ \\
\hline \multicolumn{8}{|c|}{ Temperature depression } \\
\hline TDcs-4-67 & c4B15449 & 124.4 & 3.086 & -0.2987 & 0.095 & Tх642 & Cs \\
\hline TDcs-6-12 & c6F7661 & 41.5 & 3.156 & -0.3039 & 0.103 & Tx642 & $\mathrm{Cs}$ \\
\hline TDcs-6-14 & c6B8118 & 47.2 & 4.0535 & -0.3528 & 0.1353 & Tх642 & Cs \\
\hline TDcs-8-8 & c8F2049 & 42.3 & 4.8263 & 0.3807 & 0.1609 & Tx7000 & $\mathrm{Cs}$ \\
\hline TDwl-10-33 & c10B3717 & 52.6 & 4.3184 & -0.3481 & 0.1654 & Tx642 & Wl \\
\hline TDwl-10-34 & c10B4064 & 60.3 & 5.5191 & -0.3813 & 0.1967 & Tx642 & Wl \\
\hline TDwl-10-42 & c10B11157 & 68.6 & 3.3116 & -0.278 & 0.1118 & Tх642 & Wl \\
\hline TDwl-10-54 & $\mathrm{c} 10 \mathrm{~F} 12818$ & 98.5 & 3.5743 & 0.2964 & 0.1308 & Tx7000 & Wl \\
\hline TDwl-10-58 & c10B13413 & 108.2 & 4.9324 & 0.3085 & 0.1578 & Tx7000 & $\mathrm{Wl}, \mathrm{Cx}$ \\
\hline TDcx-4-44 & c4B13239 & 93.5 & 5.4034 & 0.4575 & 0.1647 & Tx7000 & $\mathrm{Cx}$ \\
\hline TDcx-6-48 & c6F12702 & 98.8 & 3.2203 & -0.4713 & 0.1023 & Tx642 & $\mathrm{Cx}$ \\
\hline TDcx-8-9 & c8B7863 & 45 & 4.2133 & 0.454 & 0.1498 & Tx7000 & $\mathrm{Cx}$ \\
\hline TDcx-8-15 & c8F9646 & 55.8 & 6.2923 & 0.5053 & 0.1964 & Tx7000 & $\mathrm{Cx}$ \\
\hline \multicolumn{8}{|c|}{ Flowering time (days to flowering) } \\
\hline FTcx-8-15 & c8B9861 & 55.8 & 3.0584 & 5.4434 & 0.1176 & Tx7000 & $\mathrm{Cx}$ \\
\hline FTcx-10-35 & $\mathrm{c} 10 \mathrm{~F} 4552$ & 61.4 & 3.4715 & 4.9952 & 0.0812 & Tx7000 & $\mathrm{Cx}, \mathrm{Gh}$ \\
\hline
\end{tabular}

${ }^{a}$ QTL symbols: the first two upper case letters are the abbreviated traits (WL Wax load, TD canopy temperature depression, $F T$ flowering); the lower-case letters represent location ( $c s$ College Station, $c x$ Corpus Christi, $w l$ Weslaco); middle number is the chromosome number, and the last number in the marker number. Environment detected: $C s$ College Station, $C x$ Corpus Christi, $G h$ greenhouse, $W l$ Weslaco 

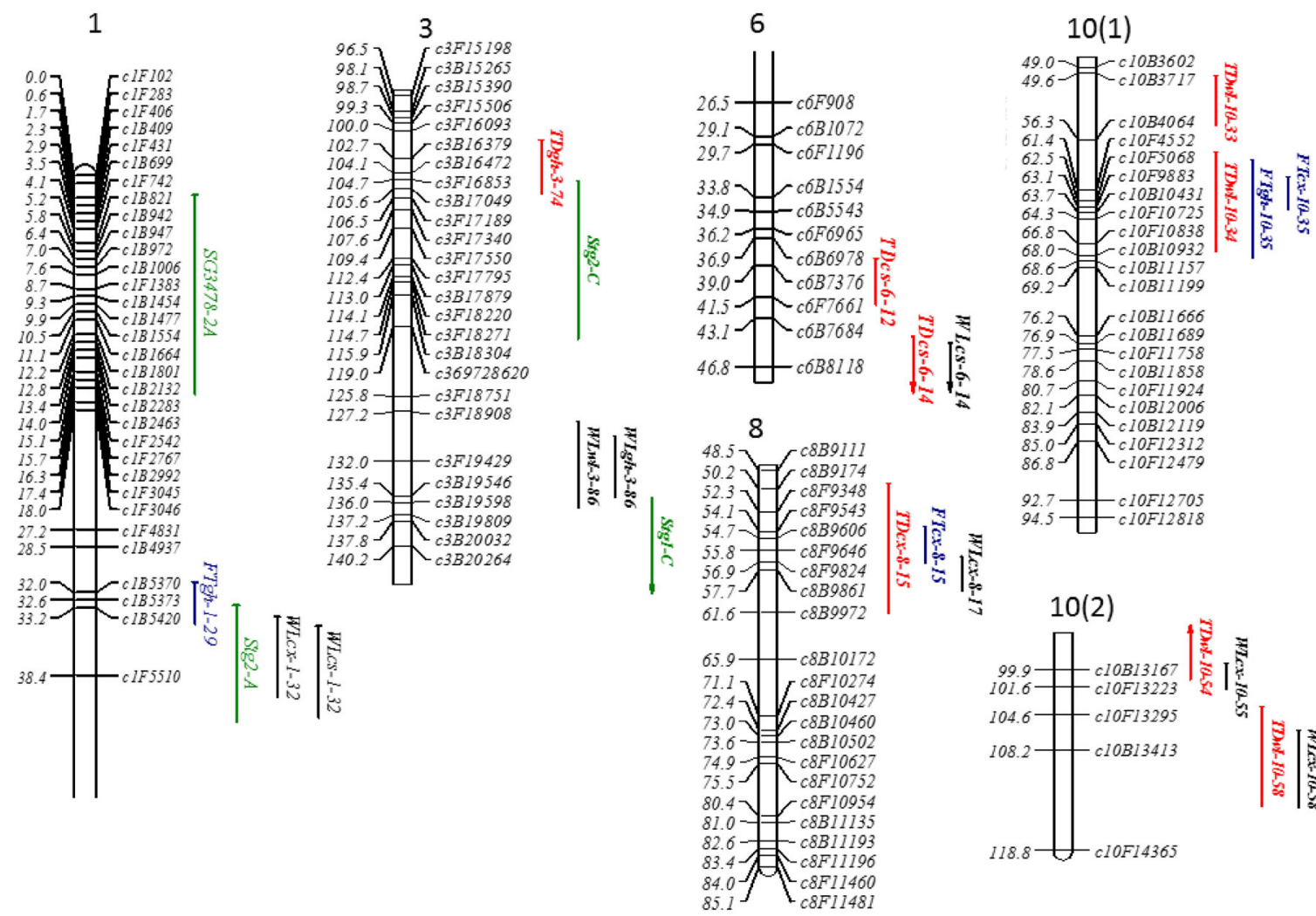

Fig. 2 Graphical representation of linkage groups on chromosome segments. Segments of chromosomes 1, 3, 6, 8 and 10 are represented. The numbers on top of each center bar represents the chromosome number. Numbers to the left are genetic distances in cM. To the right are the QTL bars where the capital letters represent the trait identity: $T D$ canopy temperature depression, FT flowering time, WL epicuticular wax load, and $S$ staygreen. Mapping was done in QTL Cartographer V2.5 and the drawings and QTL bars organized in MapChart V2.2 graphical interface. The QTL for WL, TD and FT have been

with the other Stg loci reported earlier. On SBI-01, we detected a QTL for leaf wax (WLcs-1-32) at $38.4 \mathrm{cM}$ that shared the same maker position with WLcx-1-32, explaining $12.9 \%$ of the phenotypic variance. This QTL overlapped Stg2-A at marker $c 1 F 5510$ (Fig. 2). Stg1-C, centered at marker $c 3 B 20264$ at $140.2 \mathrm{cM}$ (with interval 135.0-142.5 cM) on SBI-03 was in the same genomic region as two QTL for wax (WLgh-3-86 and $W L w l-3-86)$, centered at $135.4 \mathrm{cM}$.

On average, the mean wax and TD phenotypic contribution was increased by the Tx7000 allele with one exception (Fig. 3a, b). The QTL WLcs-132 locus had the positive allele for wax from Tx642.

overlaid on Stg QTL previously mapped by others as cited in the methods. QTL symbols: the first two upper case letters are the abbreviated traits (WL Wax load, $T D$ canopy temperature depression, FT flowering); the lower-case letters represent location (cs College Station, cx Corpus Christi, wl Weslaco); middle number is the chromosome number, and the last number in the marker number. Environment detected: Cs College Station, $C x$ Corpus Christi, Gh greenhouse, Wl Weslaco. $\mathrm{n}=100$ RILs from Tx642 X Tx7000

Annotated genes in the locus pleiotropic to wax, staygreen and flowering time

Two QTL for wax detected in two stress environments (College Station and Corpus Christi) coincided with Stg2-A and a QTL for flowering time. The genomic region bound by the overlapping QTL contained a gene cluster with a combined span of $\sim 500 \mathrm{~kb}$ close to the centromeric region of chromosome 1 (1:13139511 to 13616615$)$. The annotated genes identified in this locus (Fig. 3) include those that function in stress signaling and response (DRIP, CYP, MYB), photosynthesis and metabolite synthesis (CYP, MYB), transport (GGT, NSF) and reproduction 


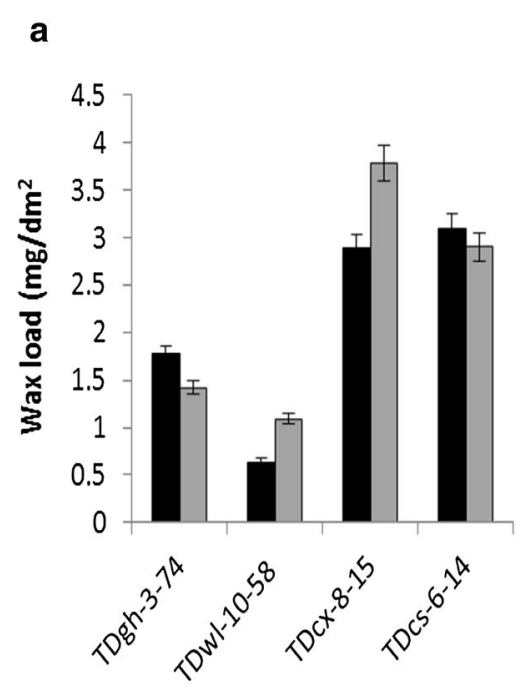

Fig. 3 The mean genotypic effects of QTL for epicuticular wax load (WL, a) and QTL for canopy temperature depression (TD, b). Only a sample of the reported QTL that showed significant

(FKL, CIPK4, KAN) (Table 4). This is a region of very low recombination, suggesting that the locus WLcs-1-32 is likely inherited as a cluster of linked genes. A summary of selected genes in the other nonstaygreen regions of QTL overlaps is shown in Table 2.

Analysis of the pleiotropic marker sequence and the flanking sequences

The 72 bp clF5510 marker is a multifunctional Cisacting regulatory module consisting of core promoter and enhancer elements, and transcription factor elements known to regulate various stress-, light-responsive-, reproductive- and seed development-associated genes in various plants (Tables 5, 6). Notable elements include the CATT-motif, a ubiquitous Cis-acting element in plants, and the TATA-box, the core promoter motif in transcription. The CATT-motif was represented in Zea mays, while the TATA-box was represented in six plant species. On both strands (Table 5) is an RY Cis-motif, a promoter element which is conserved in many seed-specific promoters that control gene expression during late embryogenesis and seed development (Reidt et al. 2000).

Within $2 \mathrm{~kb}$ of the peak marker $c 1 F 5510$ is a putative plant-specific (Baranowskij et al. 1994) and multifunctional (Rose et al. 1999) MYB DNA-binding domain

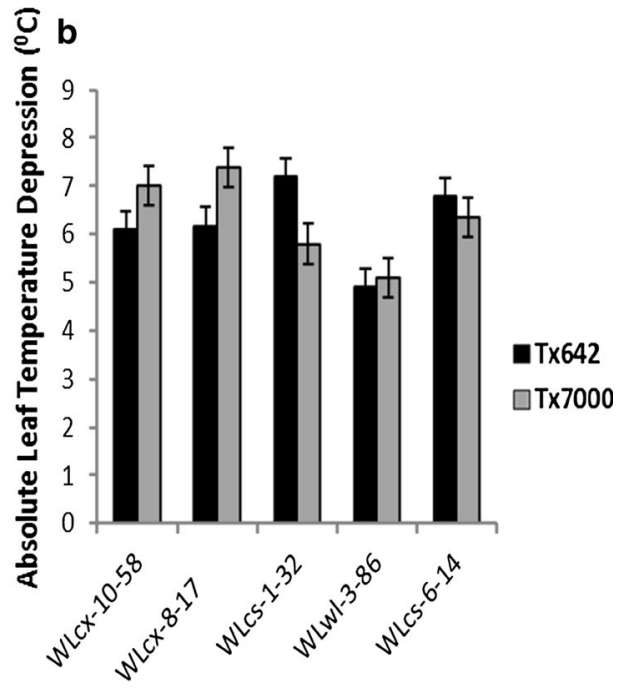

overlap with those of temperature, wax or staygreen, have been shown. The parental sources of the alleles are identified by Tx642 and Tx7000. $\mathrm{n}=100$

(ID: IPR006447), and just $\sim 500$ bp upstream of the locus peak marker is a MYB binding motif (TAACTG) involved in drought-inducibility (Table 4). The flanking sequences ( $1 \mathrm{~kb}$ on both sides of $c 1 F 5510$ ) consist of Cis-acting core regulatory elements in promoter and enhancer regions (TATA-box, TA-rich region, G-box and CAAT-box), light responsive (L-box, G-box), and Methyljasmonate, MeJA-responsivenes (CGTCA-motif) (Table 6). These observations suggest that the marker c1F5510 represents a region that may be important in stress response and reproduction.

\section{Discussion}

Wax and absolute temperature depression show a positive correlation which is both genotypically and environmentally dependent

In all the study environments, genotypes with a larger mean WL had a larger absolute TD than genotypes with smaller WL values. Mondal et al. (2015) also reported that under hot and dry conditions, leaf wax accumulation is positively correlated with the ability of a plant to keep itself cool. Stress may also have elicited a stronger wax response as a stress mitigation phenotype. Thus, large temperature may have combined with drought to elicit the stronger wax to TD 


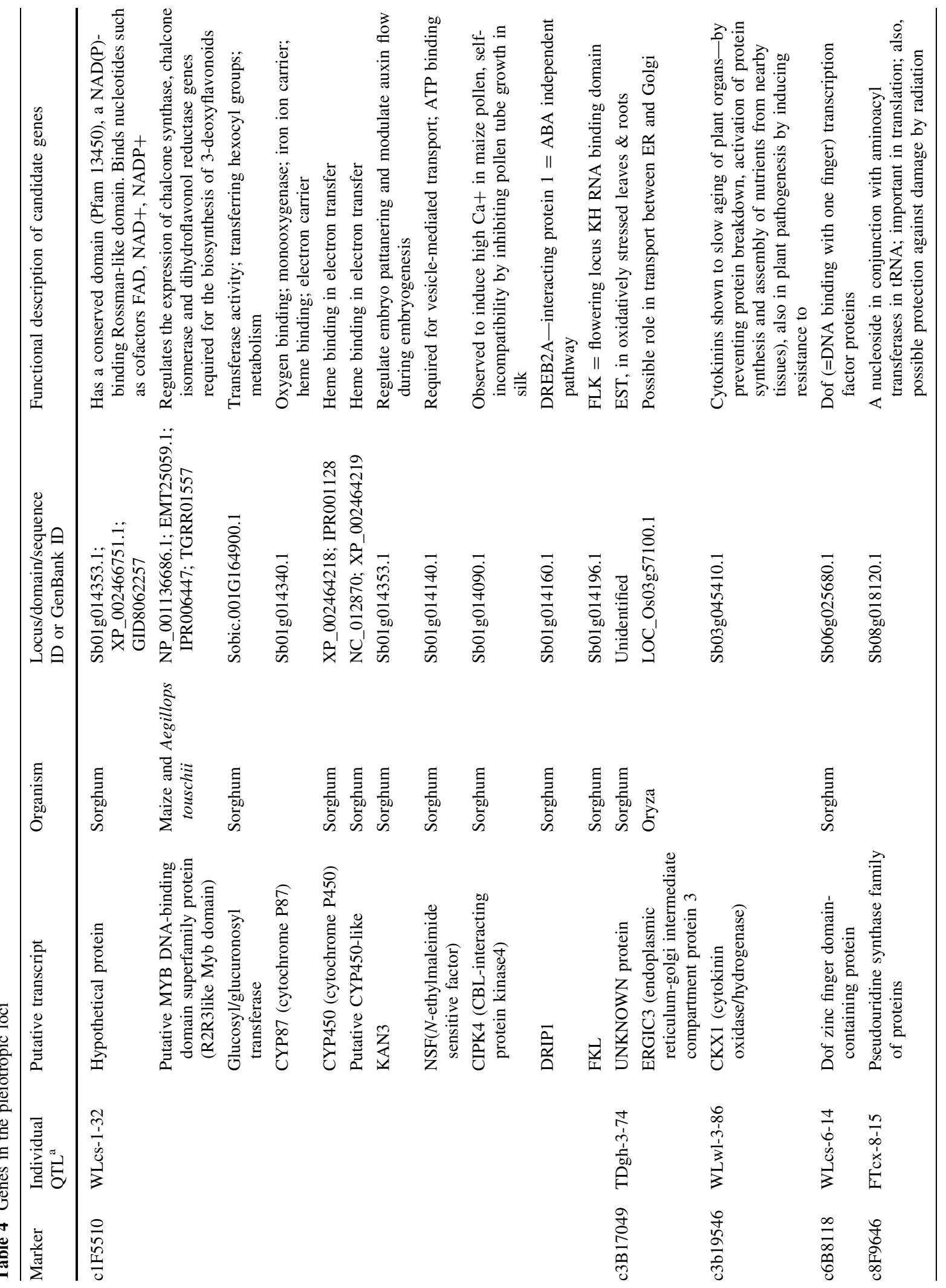


relationship in Corpus Christi $\left(\mathrm{r}^{2}=0.46\right)$ compared to the other field conditions. However, under cooler temperatures, the relationship is less obvious. For example, there was no correlation in Weslaco but a large correlation in the greenhouse condition. Weslaco and the greenhouse are two substantially different environments and the variation in response may in fact be due to the differences in confounding environmental factors given the significant variances of genotype, and $\mathrm{G} \times \mathrm{E}$ interaction for both WL and TD (Table 1). However, additional evaluations are needed to fully dissect the factors involved.

Assessment of the genotypic correlation between WL and TD showed values ranging from 0.08 in the cooler, irrigated Weslaco field environment to a high of 0.88 in the heat-stressed College Station. This trend was very similar to the proportion of observed phenotypic correlation between the two traits that is due to genetics in each of the environments. The byenvironment broad-sense heritability also showed a pattern that was similar between the two traits. These observations suggest that canopy temperature regulation is partly surrogate to the variation in WL and thus changing heritability of WL may also lead to corresponding changes in heritability of TD as a secondary, linked trait. Even though the magnitude and sign of phenotypic and genetic correlations observed may sometimes be unrelated (Réale and Roff 2001; Hill et al. 2008), our observations suggest that wax may play a role in conditioning canopy temperature and that WL has the potential to be used as an important phenotype for improving plant canopy-related stress tolerance goals.

Collocation of QTL for staygreen and those for leaf wax and temperature depression

Most Stg QTL are located on SBI-02 and SBI-05 (Xu et al. 2000; Haussmann et al. 2002; Subudhi et al. 2000; Crasta et al. 1999). In this study, only the staygreen QTL Stg 1, Stg2, Stg3 and Stg4 were considered as Stg loci (Xu et al. 2000; Crasta et al. 1999). No QTL for WL, TD and flowering were detected on these chromosomes in this study. In fact, only staygreen QTL on SBI-01 (Stg2) and SBI-03 (Stg1 and Stg2) co-located with the QTL for any of these traits have been reported in this study (Fig. 2).

Two QTL for wax WLwl-3-86 and WLgh-3-86 centered at $135.4 \mathrm{cM}$ (marker c3F19429) shared a 


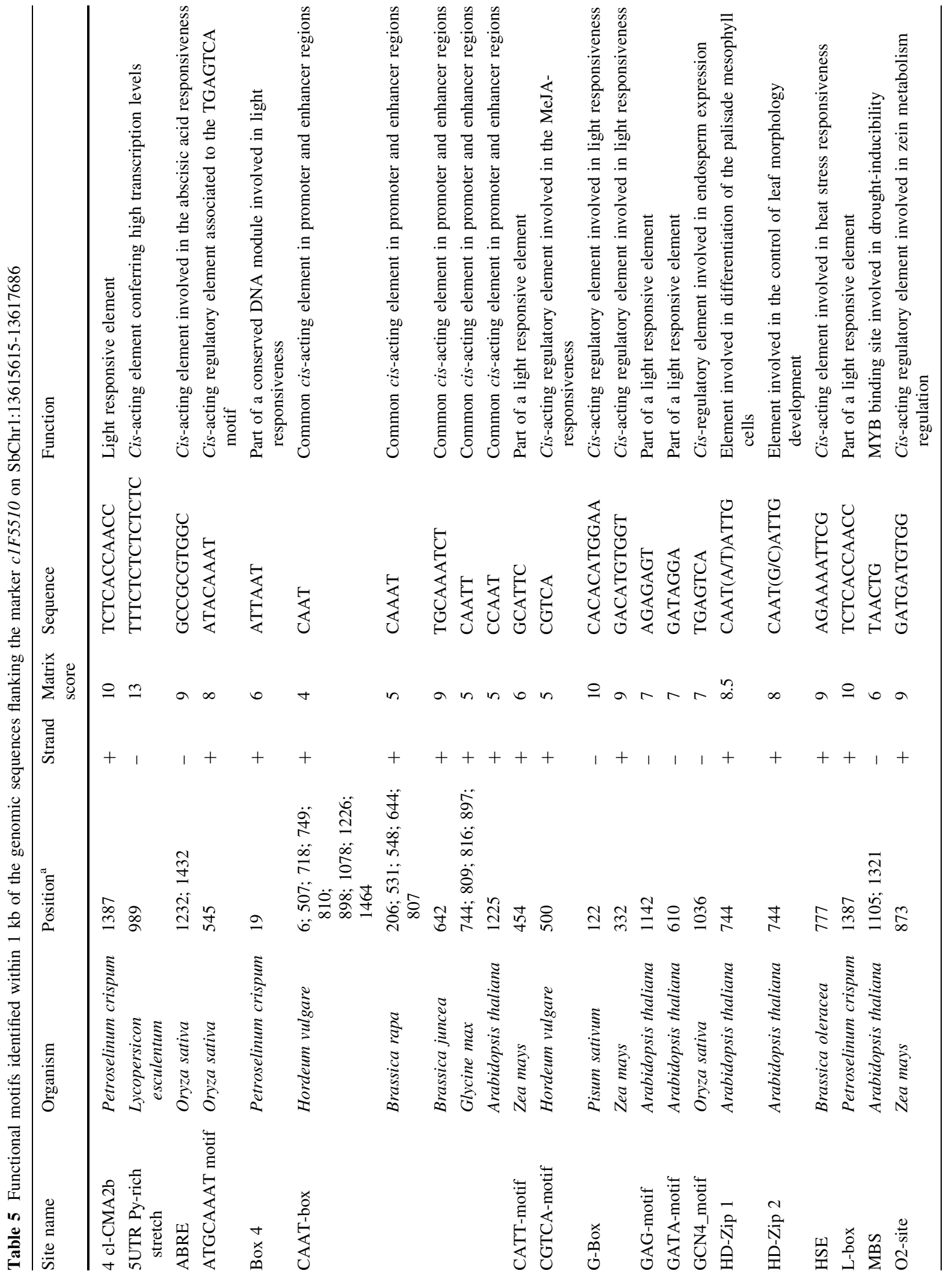




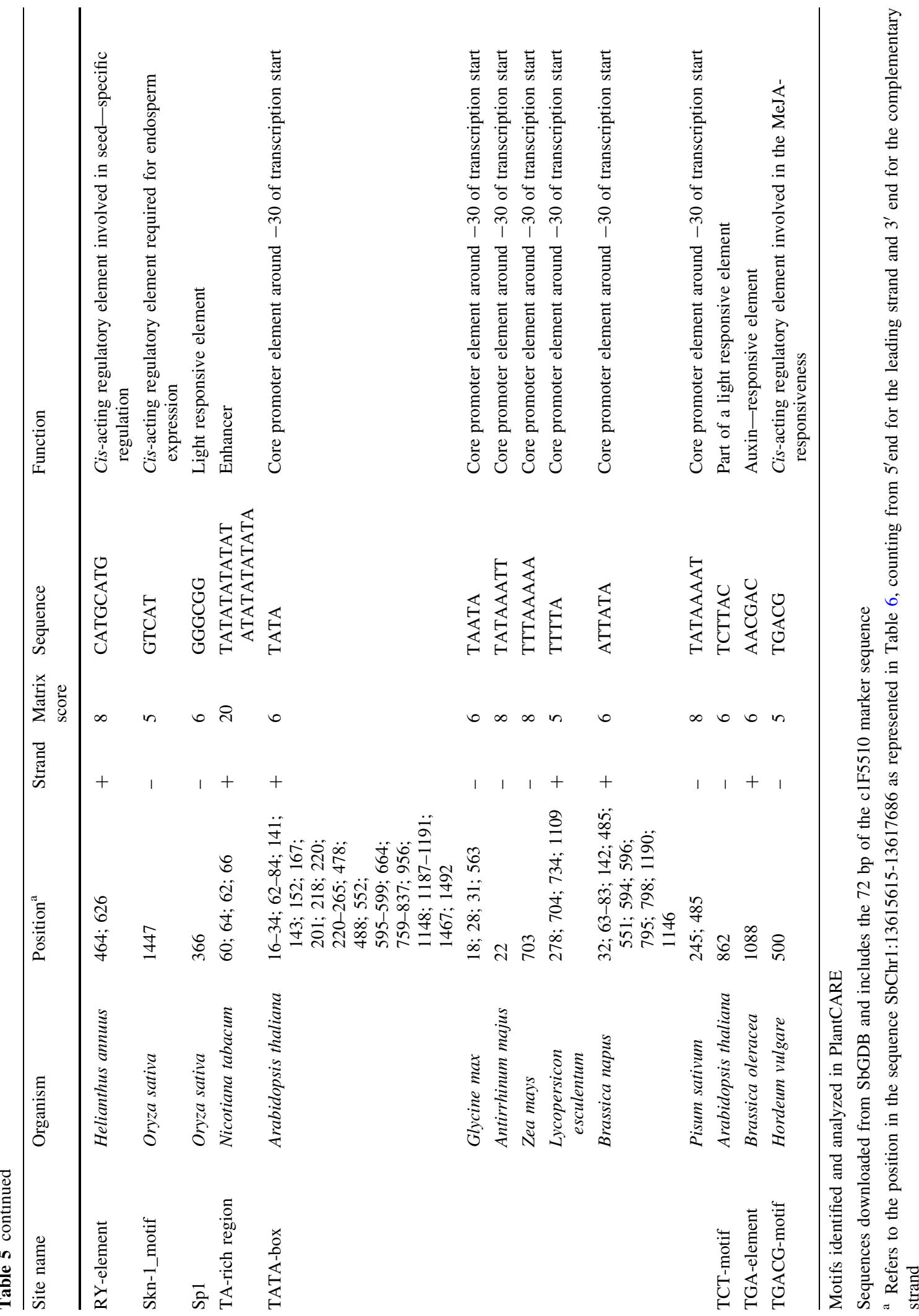




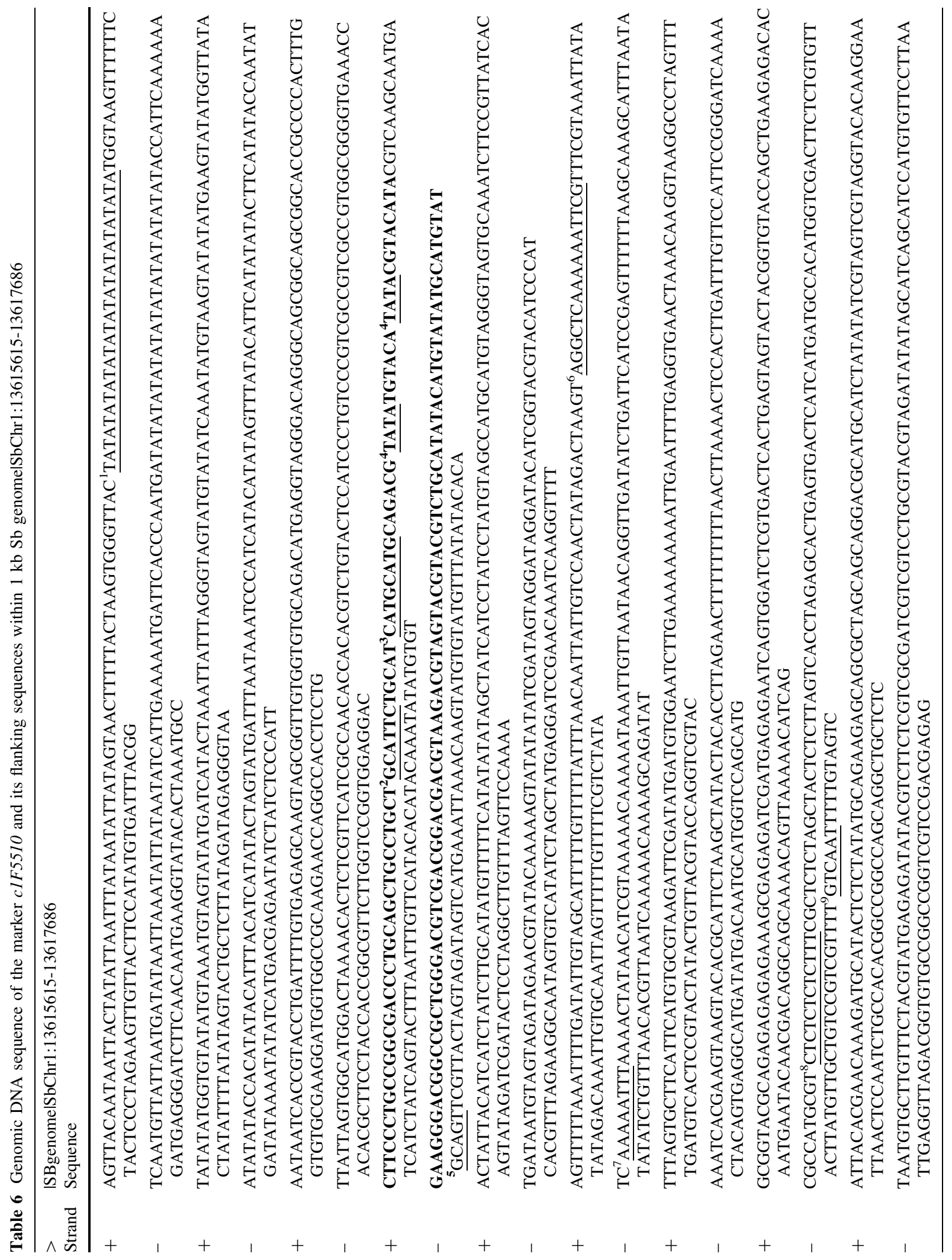




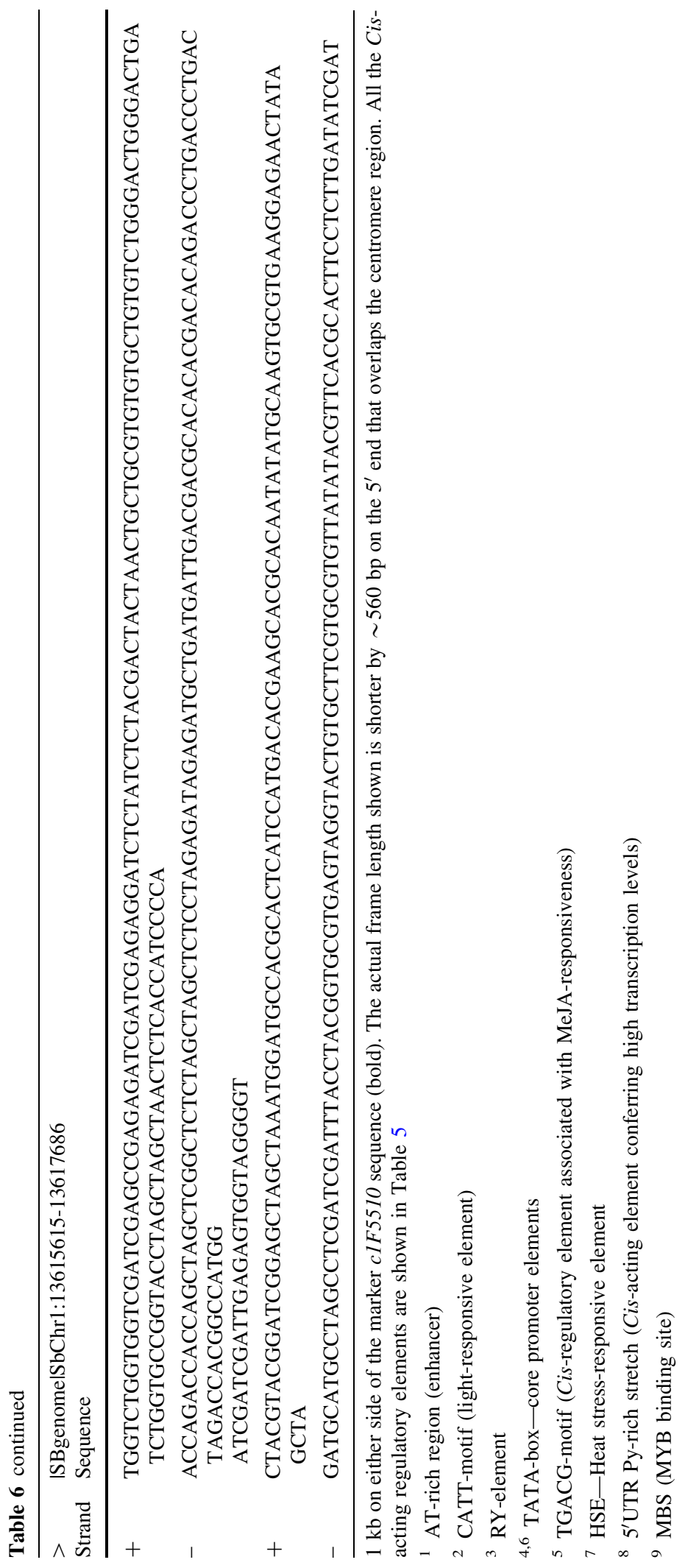


$0.5 \mathrm{cM}$ region with a Stg1 QTL $($ Stg1-C) at $140.2 \mathrm{cM}$ (marker c3B20264) on SBI-03. However, the significant wax alleles were from the non-staygreen parent Tx7000, suggesting that wax accumulation at this locus is independent of Stg1. Among all the stable staygreen QTL, Stgl QTL has been reported as one of the most important in drought tolerance after Stg2 (Crasta et al. 1999; Sanchez et al. 2002; Xu et al. 2000).

The QTL for wax, WLcs-6-14 (12\% PVE) overlapped QTL for canopy temperature TD-6-14 (13.5\% PVE) on SBI-06. Both were detected in College Station and the alleles were from the staygreen parent Tx642. The Tx642 alleles for the QTL WLcs-6-14 was associated with a slightly larger but not significantly different wax load value compared to the contribution by Tx7000 alleles. A similar observation was made for the QTL TDcs-6-14 for canopy temperature depression. However, the QTL WLcs-1-32 (12.9\% PVE) for wax load which overlapped the QTL for staygreen Stg2-A contributed to a significantly larger wax load phenotype associated with the Tx642 alleles compared the Tx7000 alleles. This QTL was mapped to a single marker $c 1 F 5510$ with another QTL for wax (WLcx-132 ) at $38.4 \mathrm{cM}$ on the short arm of SBI-01 near the centromeric region. Both QTL were detected in both the high temperature-stressed but irrigated College Station and in the drought-treated Corpus Christi. These QTL were mapped very close to a QTL ( $F T-1$ 29) detected in the greenhouse for flowering time. These observations suggest that wax load and the staygreen-associated regulatory factors may be pleiotropic.

Furthermore, the detection and collocation of the Stg loci with the genomic loci for wax may indicate that the Stg QTL overlap in time with those conferring wax-related tolerances at anthesis. This implies that that the expression of the staygreen trait may be initiated earlier than traditionally thought. Such an inference is strengthened by the conclusions based on a few recent reports on possible pre-anthesis staygreen-specific expressions (Johnson et al. 2015; Kassahun et al. 2010; Burke et al. 2010).

A tight gene cluster in the pleiotropic locus associated with wax and staygreen

Diverse genes associated with stress signaling and response (DRIP, CYP, MYB), photosynthesis and metabolite synthesis (CYP, MYB), transport (GGT,
NSF) and reproduction (FKL, CIPK4, KAN) form a gene cluster within $\sim 500 \mathrm{~kb}$ region (Table 3 ) within the locus WLcs-1-32, close to the centromere (Fig. 2). This is a region of very low recombination which suggests this locus is inherited as a cluster of linked genes.

Members of the MYB family of proteins are known to intervene in many biological processes including reproductive processes, photosynthesis and stress response, modulation of root growth and development and salicylic acid biosynthesis (Lee and Suh 2015). In the locus WLcs-1-32 resides an orthologue of a plantspecific $M y b$ domain (InterPro ID: IPR006447) which is associated with transcription regulation of stress responsive genes (Rose et al. 1999; Baranowskij et al. 1994), leaf coloration (Gao et al. 2013), floral meristem differentiation (Moreau et al. 2016) and transcriptional activation (Baranowskij et al. 1994). Other studies have shown that a $M y b$ family member, MYB94 for instance, is a transcription factor that induces both stomatal closure and cuticular wax biosynthesis by upregulating directly cuticular wax biosynthetic enzyme genes such as ketoacyl synthases (KAS), the bifunctional wax ester synthase/diacylglyceral acyltransferase (WSD1), alcohol forming fatty acyl-CoA reductase (FAR) and ECERIFERUM (CER1 and CER3) (Wang et al. 2014; Lee and Suh 2015). Lee and Suh (2015) have also shown that in drought-treated Arabidopsis, MYB96-1D and MYB96-1 upregulates 19 genes involved in wax biosynthesis, including a long-chain acyl-CoA synthase (LACS3) and in transport including an $\mathrm{ABC}$ transporter (ABCG11/WBC11/COF1). Others include lipid transfer proteins (LTP) (Seo and Park 2011). Additive effects were recently reported between MYB94 and MYB96 in the activation of cuticular wax biosynthesis in Arabidopsis (Lee et al. 2016). The presence of the a MYB binding site (MBS, TAACTG) involved in droughtinducibility and the Drought Responsive Element Binding, DREB2A-Interacting Protein (DRIP1) within WLcs-1-32 suggests that this QTL may be involved in drought-related responses. Further, the locus contains a second major domain, the Rossemann-like NAD_binding_8 (PFAM13450) which is a structural motif in the hypothetical protein SORBIDRAFT_01g014353 (orthologue Si037864 m.g) (Table 3), which binds nucleotides such as cofactors FAD, NAD + and $\mathrm{NADP}+$ that are important in electron transfer in photosystems. Together with the presence of other Cisacting elements in the promoter and enhancer regions 
(Table 6), we suggest that the locus WLcs-1-32 may be important in the transcription of stress responsive genes, which may include those regulating wax, leaf pigmentation and photosynthetic processes.

Similarly, the pleiotropic effect of WLcs-1-32 can also be attributed to the CYP family of proteins in this locus. For instance, the CYP87 is reported to directly be associated with oxygen and electron transfer by acting in heme binding and as iron carrier during photosynthesis (Werck-Reichhart et al. 2002), while Phytochorme450 (P450)-like proteins are involved in stress and defensive compounds signaling cascades such as for plant hormones and fatty acids, cutin synthesis and functional plant pigments (WerckReichhart et al. 2002; Degtyarenko 1995). A Cytochrome P450 family member, CYP704B2, has been shown to catalyze the $\omega$-hydroxylation of fatty acids and is required for anther cutin biosynthesis and pollen exine formation in rice ( $\mathrm{Li}$ et al. 2010). A related protein, CYP96A15, which is also upregulated by a $M y b$ gene, is a functional orthologue of Mid-chain Alkane Hydroxylase (MAH1), which converts alkanes to $1^{0}$ alcohols and $1^{0}$ alcohols to ketones in the $1^{0}$ alcohol pathway in wax biosynthesis (Seo and Park 2011). Other genes residing in the locus intervene directly in the reproductive processes (CIPKL4, KAN3, FKL), drought (DRIP1) and vesicular transport (NSF, GGT).

The marker $c 1 F 5510$, together with its $2 \mathrm{~kb}$ flanking sequences, is a region of Cis-regulatory module (CRM). Transcriptional regulation is usually mediated by the interaction of multiple transcription factors (TFs) with their respective specific Cis-regulatory elements (CREs) in the CRM in the DNA (Niu et al. 2014). Taken together, we suggest that the locus WLcs-1-32 may have a role in the transcriptional regulation of wax biosynthesis and functional staygreen, among other functions during inflorescence and anthesis in Sorghum under stress.

\section{Conclusion}

This study investigated the genetic overlap between QTL for staygreen, epicuticular leaf wax and canopy temperature depression. We have also reported that field stress conditions may elicit a stronger genetic correlation between epicuticular wax load and canopy temperature depression than the unstressed field conditions. Thus, selecting for wax load may improve the proportion of observed phenotypic correlation which is due to genetics. This observation is strengthened by the fact that a number of traits collocated to the same QTL. The results suggest a possible strong linkage between QTL for epicuticular leaf wax and canopy temperature depression and that wax load influences plant canopy temperature. QTL associated with flowering time were also detected. The overlap between a QTL for epicuticular leaf wax and a QTL for staygreen (Stg2-A) showed that the alleles for larger epicuticular wax load was from the postanthesis staygreen parent Tx642. This was different from the other QTL for larger epicuticular wax load which were associated with the pre-anthesis drought tolerant Tx7000. The onset of expression of the different staygreen loci may vary with stress level. Our results suggest that the genes controlling the staygreen trait may be linked with those controlling leaf waxes to influence a plant response to drought and heat stress at the reproductive stage. Further studies of these major QTL are recommended.

\section{Compliance with ethical standards}

Conflict of interest The authors declare that they have no conflict of interest.

Open Access This article is distributed under the terms of the Creative Commons Attribution 4.0 International License (http:// creativecommons.org/licenses/by/4.0/), which permits unrestricted use, distribution, and reproduction in any medium, provided you give appropriate credit to the original author(s) and the source, provide a link to the Creative Commons license, and indicate if changes were made.

\section{References}

Balota M, Payne WA, Evett SR, Lazar MD (2007a) Canopy temperature depression sampling to assess grain yield and genotypic differentiation in winter wheat. Crop Sci 47(4):1518-1529

Balota M, Payne WA, Evett SR, Lazar MD (2007b) Canopy temperature depression sampling to assess grain yield and genotypic differentiation in winter wheat. Crop Science 47(4):1518-1529

Baranowskij N, Frohberg C, Prat S, Willmitzer L (1994) A novel DNA binding protein with homology to Myb oncoproteins containing only one repeat can function as a transcriptional activator. EMBO J 13(22):5383

Baum LE, Petrie T (1966) Statistical inference for probabilistic functions of finite state Markov chains. Ann Math Stat. doi:10.1214/aoms/1177699147 
Bernardo R (2002) Breeding for quantitative traits in plants, vol 576.5 B523. Stemma Press, Woodbury

Blum A (2005) Drought resistance, water-use efficiency, and yield potential—are they compatible, dissonant, or mutually exclusive? Aust J Agric Res 56(11):1159-1168. doi:10.1071/AR05069

Burke J, Franks C, Burow G, Xin Z (2010) Selection system for the stay-green drought tolerance trait in sorghum germplasm. Agron J 102(4):1118-1122

Cartegni LWJ, Zhu Z, Zhang MQ, Krainer AR (2003) ESEfinder: a web resource to identify exonic splicing enhancers. Nucleic Acid Res 31(13):3568-3571

Clarke JM, Richards RA (1988) The effects of glaucousness, epicuticular wax, leaf age, plant height and growth environment on water loss rates of exiced wheat leaves. Can J Plant Sci 68(4):975-982. doi:10.4141/cjps88-118

Consortium U (2014) UniProt: a hub for protein information. Nucleic Acids Res. doi:10.1093/nar/gku989

Crasta OR, Xu WW, Rosenow DT, Mullet J, Nguyen HT (1999) Mapping of post-flowering drought resistance traits in grain sorghum: association between QTLs influencing premature senescence and maturity. Mol Gen Genet 262(3):579-588. doi:10.1007/s004380051120

Degtyarenko KN (1995) Structural domains of P450-containing monooxygenase systems. Protein Eng 8(8):737-747. doi:10.1093/protein/8.8.737

Ebercon A, Blum A, Jordan WR (1977) A rapid colorimetric method for epicuticular wax contest of sorghum leaves. Crop Sci 17(1):179-180. doi:10.2135/cropsci1977. 0011183X001700010047x

Falconer DS, Mackay TF, Frankham R (1996) Introduction to quantitative genetics. Trends in genetics, 4th edn. 12(7):280

Fan TB, Balta M, Rudd J, Payne WA (2005) Canopy temperature depression as a potential selection criterion for drought resistance in wheat. Agric Sci China 4(4):8

Gao Z, Liu C, Zhang Y, Li Y, Yi K, Zhao X, Cui M-L (2013) The promoter structure differentiation of a MYB transcription factor RLC1 causes red leaf coloration in empire red leaf cotton under light. PLoS ONE 8(10):e77891

Harris K, Subudhi PK, Borrell A, Jordan D, Rosenow DL, Nguyen H, Klein P, Klein R, Mullet J (2007) Sorghum stay-green QTL individually reduce post-flowering drought-induced leaf senescence. J Exp Bot 58(2):327-338. doi:10.1093/jxb/erl225

Haussmann BH, Mahalakshmi VM, Reddy BR, Seetharama NS, Hash CH, Geiger HG (2002) QTL mapping of stay-green in two sorghum recombinant inbred populations. Theor Appl Genet 106(1):133-142. doi:10.1007/s00122-002-1012-3

He Y, Fukushige H, Hildebrand DF, Gan S (2002) Evidence supporting a role of jasmonic acid in arabidopsis leaf senescence. Plant Physiol 128(3):876-884

Hill WG, Goddard ME, Visscher PM (2008) Data and theory point to mainly additive genetic variance for complex traits. PLoS Genet 4(2):e1000008

Illumina.com (2014) doi:10.1016/j.fertnstert.2014.01.051

Johnson SM, Cummins I, Lim FL, Slabas AR, Knight MR (2015) Transcriptomic analysis comparing stay-green and senescent Sorghum bicolor lines identifies a role for proline biosynthesis in the stay-green trait. J Exp Bot. doi:10.1093/ jxb/erv405
Kassahun B, Bidinger F, Hash C, Kuruvinashetti M (2010) Staygreen expression in early generation Sorghum bicolor (L.) Moench QTL introgression lines. Euphytica 172(3):351-362. doi:10.1007/s10681-009-0108-0

Kaufman L, Rousseeuw PJ (2009) Finding groups in data: an introduction to cluster analysis, vol 344. Wiley, Hoboken

Keener ME, Kircher PL (1983) The use of canopy temperature as an indicator of drought stress in humid regions. Agric Meteorol 28(4):339-349. doi:10.1016/0002-1571(83)90010-9

Kumari M, Singh VP, Tripathi R, Joshi AK (2007) Variation for staygreen trait and its association with canopy temperature depression and yield traits under terminal heat stress in wheat production in stressed environments. In: Buck HT, Nisi JE, Salomón N (eds) Developments in Plant Breeding, vol 12. Springer, Netherlands, pp 357-363. doi:10.1007/14020-5497-1_44

Lee SB, Suh MC (2015) Advances in the understanding of cuticular waxes in Arabidopsis thaliana and crop species. Plant Cell Rep 34(4):557-572

Lee SB, Kim HU, Suh MC (2016) MYB94 and MYB96 additively activate cuticular wax biosynthesis in Arabidopsis. Plant Cell Physiol. doi:10.1093/pcp/pcw147

Li H, Pinot F, Sauveplane V, Werck-Reichhart D, Diehl P, Schreiber L, Franke R, Zhang P, Chen L, Gao Y (2010) Cytochrome P450 family member CYP704B2 catalyzes the $\omega$-hydroxylation of fatty acids and is required for anther cutin biosynthesis and pollen exine formation in rice. Plant Cell 22(1):173-190

Lim PO, Nam HG (2005) The molecular and genetic control of leaf senescence and longevity in arabidopsis. Curr Top Dev Biol 67:50-85

Lim PO, Woo HR, Nam HG (2003) Molecular genetics of leaf senescence in arabidopsis. Trends Plant Sci 8(6):272-278

Maes HH, Sullivan PF, Bulik CM, Neale MC, Prescott CA, Eaves LJ, Kendler KS (2004) A twin study of genetic and environmental influences on tobacco initiation, regular tobacco use and nicotine dependence. Psychol Med 34(7):1251-1261

Magali Lescot PDh, Thijs Gert, Marchal Kathleen, Moreau Yves, Van de Peer Yves, Rouz Pierre, Rombauts Stephane (2002) PlantCARE, a database of plant cis-acting regulatory elements and a portal to tools for in silico analysis of promoter sequences. Nucleic Acids Res 30(1):325-327

McLaughlin JE, Boyer JS (2004) Sugar-responsive gene expression, invertase activity, and senescence in aborting maize ovaries at low water potentials. Ann Bot 94(5):675-689. doi:10.1093/aob/mch193

Mondal S, Mason R, Huggins T, Hays D (2015) QTL on wheat (Triticum aestivum $\mathrm{L}$.) chromosomes $1 \mathrm{~B}, 3 \mathrm{D}$ and $5 \mathrm{~A}$ are associated with constitutive production of leaf cuticular wax and may contribute to lower leaf temperatures under heat stress. Euphytica 201(1):123-130. doi:10.1007/ s10681-014-1193-2

Moreau F, Thévenon E, Blanvillain R, Lopez-Vidriero I, Franco-Zorrilla JM, Dumas R, Parcy F, Morel P, Trehin C, Carles CC (2016) The Myb-domain protein ULTRAPETALA1 INTERACTING FACTOR 1 controls floral meristem activities in Arabidopsis. Development 143(7):1108-1119

Morishige DT, Klein PE, Hilley JL, Sahraeian SME, Sharma A, Mullet JE (2013) Digital genotyping of sorghum-a 
diverse plant species with a large repeat-rich genome. BMC Genom 14(1):448

Niu M, Tabari ES, Su Z (2014) De novo prediction of cisregulatory elements and modules through integrative analysis of a large number of ChIP datasets. BMC Genom 15(1):1047. doi:10.1186/1471-2164-15-1047

Noh Y-S, Amasino RM (1999) Identification of a promoter region responsible for the senescence-specific expression of SAG12. Plant Mol Biol 41(2):181-194

O'Neill M (2010) ANOVA \& REML: a guide to linear mixed models in an experimental design context. Statistical Advisory \& Training Service Pty Ltd

Ott L, Longnecker M (2010) An introduction to statistical methods and data analysis. Brooks/Cole, Pacific Grove

Paynter R (1981) Modification of the Beer-Lambert equation for application to concentration gradients. Surf Interface Anal 3(4):186-187

Quail MA, Swerdlow H, Turner DJ (2001) Improved protocols for the illumina genome analyzer sequencing system. Current protocols in human genetics. Wiley, Hoboken. doi:10.1002/0471142905.hg1802s62

Quirino BF, Noh Y-S, Himelblau E, Amasino RM (2000) Molecular aspects of leaf senescence. Trends Plant Sci 5(7):278-282

Réale D, Roff DA (2001) Estimating genetic correlations in natural populations in the absence of pedigree information: accuracy and precision of the lynch method. Evolution 55(6):1249-1255. doi:10.1554/0014-3820(2001)055[1249: EGCINP]2.0.CO;2

Reidt W, Wohlfarth T, Ellerström M, Czihal A, Tewes A, Ezcurra I, Rask L, Bäumlein H (2000) Gene regulation during late embryogenesis: the RY motif of maturationspecific gene promoters is a direct target of the FUS3 gene product. Plant J 21(5):401-408

Reynolds M, Balota M, Delgado MIB, Amani I, Fischer RA (1994) Physiological and morphologicaltraits associated with spring wheat yield under hot, irrigated conditions. Aust J Funct Plant Biol 01(01):717-730. doi:10.1071/ PP9940717

Reynolds MP, Singh RP, Ibrahim A, Ageeb OAA, LarquéSaavedra A, Quick JS (1998) Evaluating physiological traits to complement empirical selection for wheat in warm environments. Euphytica 100(1):85-94. doi:10.1023/a: 1018355906553

Rose A, Meier I, Wienand U (1999) The tomato I-box binding factor LeMYBI is a member of a novel class of Myb-like proteins. Plant J 20(6):641-652

Rosenthal WD, Gerik TJ (1991) Flowering distribution within and among grain sorghum panicles. Crop Sci 29(4):1054-1057. doi:10.2135/cropsci1989.0011183X002900040047x

Sanchez AC, Subudhi PK, Rosenow DT, Nguyen HT (2002) Mapping QTLs associated with drought resistance in sorghum (Sorghum bicolor L. Moench). Plant Mol Biol 48(5):713-726. doi:10.1023/a:1014894130270

Scott AJ, Knott M (1974) A cluster analysis method for grouping means in the analysis of variance. Biometrics:507-512
Seo PJ, Park C-M (2011) Cuticular wax biosynthesis as a way of inducing drought resistance. Plant signal Behav 6(7):1043-1045

Smith PJ, Zhang C, Wang J, Chew SL, Zhang MQ, Krainer AR (2006) An increased specificity score matrix for the prediction of SF2/ASF-specific exonic splicing enhancers. Hum Mol Genet 15(16):2490-2508

Solovyev V (2001) Statistical approaches in eukaryotic gene prediction. Handbook of statistical genetics. Wiley, Hoboken

Solovyev V, Kosarev P, Seledsov I, Vorobyev D (2006) Automatic annotation of eukaryotic genes, pseudogenes and promoters. Genome Biol 7(1):1

Stephen F, Altschul TLM, Schäffer Alejandro A, Zhang Jinghui, Zhang Zheng, Miller Webb, Lipman David J (1997) Gapped BLAST and PSI-BLAST: a new generation of protein database search programs. Nucleic Acids Res 25:3389-3402

Stratonovich RL (1960) Conditional Markov Processes. Theory Probab Appl 5(2):156-178. doi:10.1137/1105015

Subudhi PK, Rosenow DT, Nguyen HT (2000) Quantitative trait loci for the stay green trait in sorghum (Sorghum bicolor L. Moench): consistency across genetic backgrounds and environments. Theor Appl Genet 101(5):733-741. doi:10. 1007/s001220051538

Tao YZ, Henzell RG, Jordan DR, Butler DG, Kelly AM, McIntyre CL (2000) Identification of genomic gegions associated with staygreen in sorghum by testing RILs in multiple environments. Theor Appl Genet 100(8):1225-1232. doi:10.1007/ s001220051428

Team RC (2013) R: a language and environment for statistical computing

Thomas H, Howarth CJ (2000) Five ways to stay green. J Exp Bot 51(suppl 1):329-337. doi:10.1093/jexbot/51.suppl_1. 329

Voorrips RE (2002) MapChart: software for the graphical presentation of linkage maps and QTLs. J Hered 93(1):77-78

Wachs TD, Plomin RE (1991) Conceptualization and measurement of organism-environment interaction. American Psychological Association, Washington, DC

Waitt DE, Levin DA (1998) Genetic and phenotypic correlations in plants: a botanical test of Cheverud's conjecture. Heredity 80(3):310-319

Wang Y, Wang M, Sun Y, Wang Y, Li T, Chai G, Jiang W, Shan L, Li C, Xiao E (2014) FAR5, a fatty acyl-coenzyme A reductase, is involved in primary alcohol biosynthesis of the leaf blade cuticular wax in wheat (Triticum aestivum L.). J Exp Bot. doi:10.1093/jxb/eru457

Werck-Reichhart D, Bak S, Paquette S (2002) Cytochromes P450. Arabidopsis Book. doi:10.1199/tab.0028

Wilman D, Walters RJK, Baker DH, Williams SP (1992) Comparison of two varieties of Italian ryegrass (Lolium multiflorum) for milk production, when fed as silage and when grazed. J Agric Sci 118(01):37-46. doi:10.1017/ S0021859600067988

Windows QTL Cartographer 2.5 (2011) Department of Statistics, North Carolina State University, Raleigh, NC. http:// statgen.ncsu.edu/qtlcart/WQTLCart.htm 
Worsley-Hunt R, Bernard V, Wasserman WW (2011) Identification of cis-regulatory sequence variations in individual genome sequences. Genome Med 3(10): 1

Xu W, Subudhi PK, Crasta OR, Rosenow DT, Mullet JE, Nguyen HT (2000) Molecular mapping of QTLs conferring stay-green in grain sorghum (Sorghum bicolor L. Moench). Genome 43(3):461-469. doi:10.1139/g00-003
Yao H, Guo L, Fu Y, Borsuk LA, Wen T-J, Skibbe DS, Cui X, Scheffler BE, Cao J, Emrich SJ (2005) Evaluation of five $\mathrm{ab}$ initio gene prediction programs for the discovery of maize genes. Plant Mol Biol 57(3):445-460 